\title{
ACTIVITY AND THE FORMATION OF OCULAR DOMINANCE PATCHES IN DUALLY INNERVATED TECTUM OF GOLDFISH $^{\mathbf{1}}$
}

\author{
VALERIE C. BOSS AND JOHN T. SCHMIDT ${ }^{2}$
}

Department of Biological Sciences, State University of New York at Albany, Albany, New York 12222

Received August 12, 1983; Revised June 4, 1984; Accepted June 6, 1984

\begin{abstract}
This study tested (1) whether blocking impulse activity in both eyes of fish with one tectum prevents the formation of ocular dominance patches, $(2)$ whether areas receiving a high density of innervation from one eye receive a low density from the other, and (3) whether there is an electrophysiological correlate to the anatomical patches. One tectum was removed in goldfish so that the optic nerve fibers from both eyes would compete for synaptic space in the remaining tectum. The terminal arbors from the two projections initially overlapped but by 50 to 60 days segregated into ocular dominance patches, demonstrated by labeling both projections, the normal one with horseradish peroxidase and the regenerating one with tritiated proline radioautography. Alternate sections were processed for radioautography and histochemistry. All projections were drawn by "blind" observers using a camera lucida and were fully reconstructed. Both the level of patchiness within each projection and the correspondence of patches and holes between the two projections were quantified from these reconstructions. Binocular tetrodotoxin (TTX) injections from 18 to 75 days after surgery significantly reduced patchiness, as compared to controls injected with citrate-Ringers solution. When the binocular block was continued until 95 days, segregation was still significantly reduced relative to controls. These results support a hypothesis for an activity-dependent mechanism of segregation of ocular dominance patches.

In controls but not TTX-blocked fish, there was a significant tendency for high density areas in one projection to receive a lower density projection from the other eye, and vice versa. However, the two projections were not entirely complementary. Survival of control fish for an additional 5 months resulted in more sharply defined patches but no increase in complementarity.

Recordings of field potentials evoked by shocking either optic nerve demonstrated an electrophysiological correlate to the anatomical patches in single tectal fish. Large field potentials from one eye were generally associated with small potentials from the other eye, and vice versa. When the recording sites were marked with electrolytic lesions, there was a direct and significant correlation between the magnitude of the field potentials and the density of the anatomical ocular dominance patches.
\end{abstract}

In vertebrates, it is common for the terminal arborizations of afferents from the two eyes to segregate into stripes. In the visual cortex and tectum of cats and monkeys, ocular dominance patches form as a normal part of development (Hubel and Wiesel, 1969; Wiesel et al., 1974; Rakic, 1976; LeVay et al., 1978). In lower vertebrates, stripe formation can be induced by forcing two visual projections to innervate a target which is normally innervated by only one. This has been accomplished in frogs by removing a tectum, adding an eye, or creating a compound eye (Constantine-Paton and Law, 1978; Law and Constantine-Paton, 1980, 1981; Fawcett and Willshaw, 1982), and in goldfish by removing one tectum or deflecting a bundle of fibers (Levine and Jacobson, 1975; Easter et al., 1978;

\footnotetext{
${ }^{1}$ We thank Drs. David Tieman, Suzannah Tieman, Rod Murphey, and Helmut V. B. Hirsch for useful comments, Dr. David Lotshaw for help with the video microscopy, and M. Buzzard for technical assistance. This work was supported by National Institutes of Health Grant EY03736, a State University of New York Research Foundation grant, and a Sloan Foundation Fellowship to J. T. S.

${ }^{2}$ To whom correspondence should be addressed.
}

Schmidt, 1978; Lo and Levine, 1980; Springer and Cohen, 1981; Meyer, 1982). In all cases, the pathways project in a retinotopically ordered manner onto the target area (Sharma, 1973; Easter and Schmidt, 1977) and appear to segregate into eyespecific terminal zones. When one pathway is labeled, these zones appear as rostrocaudally oriented ocular dominance stripes in the layer of optic fiber termination in the tectum. Electrophysiological correlates of such banding have been surprisingly difficult to demonstrate. Units from both eyes can still be recorded over nearly the entire tectal surface both in fish (Schmidt, 1978) and in frogs (Law and Constantine-Paton, 1981), although units from one eye are often larger than those from the other.

The goldfish retinotectal system is a good model in which to study segregation of retinal afferents. The development of stripes in goldfish is similar to that seen in the mammalian visual system, with an initial period of overlap between the two projections followed by segregation (Schmidt, 1978; Lo and Levine, 1980; Springer and Cohen, 1981). The retinotectal projection, however, is simpler than the pathway from retina to cortex in mammals because it is monosynaptic. In goldfish, the projections from the two eyes are normally completely 
crossed, so that there is no direct competition between them in the tectum. When a tectum is removed, the remaining tectal lobe is invaded by an extra set of optic fibers, and each projection sets up a retinotopically ordered map across the tectum (Sharma, 1973; Easter and Schmidt, 1977; Schmidt, 1978; Law and Constantine-Paton, 1981). The projections segregate in about 2 months' time. Since goldfish optic fibers can regenerate, stripe formation can be induced in goldfish of various sizes and ages (Levine and Jacobson, 1975; Schmidt, 1978; Springer and Cohen, 1981). The goldfish visual system is easily manipulable because the tectum is highly accessible and because the retinotectal pathway is easily labeled with proline or horseradish peroxidase (HRP).

Recent studies in kittens suggest that blocking the activity of both eyes may prevent the formation of ocular dominance patches (Stryker, 1981) and that dark rearing decreases segregation (Swindale, 1981). Previous work demonstrated that monocular deprivation in cats and monkeys reduces the width of ocular dominance patches driven by the deprived eye while increasing the width of patches driven by the experienced eye (Hubel et al., 1977; Shatz and Stryker, 1978; LeVay et al., 1980). Those studies suggest that nerve impulse activity may be involved both in the segregation of visual pathways in mammals and in the relative areas controlled by each eye.

In goldfish, Meyer (1982) reported that binocular injection of tetrodotoxin (TTX) could block the segregation of the terminals of a small bundle of fibers deflected across the midline from one tectum to another. In this study, we deflected the entire optic tract from one eye, reconstructed the labeled projections from both eyes, and quantitatively tested whether activity was necessary for the initial formation of ocular dominance patches in goldfish. We found that initial segregation was significantly reduced by binocular block of activity. We also demonstrated that high density areas in each projection corresponded to low density areas in the other projection in control but not in TTX-blocked fish, and that the segregation into ocular dominance patches was reflected in the amplitudes of field potentials elicited by optic nerve shock. Some of these observations were reported previously in abstract form (Boss and Schmidt, 1982).

\section{Materials and Methods}

Surgery. Goldfish (Carassius auratus), 8 to $10 \mathrm{~cm}$ long, were obtained from Grassyforks Fisheries (Martinsville, IN). Before surgery, the goldfish were anesthetized by immersion in $0.1 \%$ solution of tricaine methanesulfonate (TMS). The cranium was opened and the left tectum and entire forebrain were excised. The right optic nerve was deflected toward the remaining right optic tectum at the level of the optic tract. In a previous study this deflection technique seemed to produce a more complete invasion of the ipsilateral tectum (Schmidt, 1978). The left optic nerve was left intact. The cranial covering was sealed with cyanoacrylate, and the fish were revived and kept at $30^{\circ} \mathrm{C}$.

TTX injections. Repeated intraocular injections of TTX were used to block action potentials in one or both eyes. In each case, $1 \mu \mathrm{l}$ of 0.1 mM TTX (Sigma Chemical Co., St. Louis, MO, supplied in dry citrate buffer) in goldfish Ringers solution, $\mathrm{pH} 7$, was injected into the vitreous using a $10-\mu$ l Hamilton syringe. A single injection at this dosage has been shown to block activity in the eye for 2 to 2.5 days (Edwards and Grafstein, 1983; Schmidt and Edwards, 1983). Injections were repeated every other day to achieve a continuous block. Two groups of fish were injected. "Bilateral TTX" fish were injected with TTX into both eyes beginning at 18 days and continuing until sacrifice at 73 to 78 or 93 to 98 days after surgery. Eighteen days was chosen because it is approximately the time when regenerating fibers first appear in the optic lamina of the ipsilateral tectum (Springer and Cohen, 1981). Operated control animals were given $1-\mu$ l injections of citrate-Ringers solution $(0.59 \mathrm{~mm}$ citrate in goldfish Ringers solution adjusted to $\mathrm{pH} 7$ with $\mathrm{HCl}$ ) on the same schedule as the TTX injections. This is the same amount of citrate as was contained in the TTX solution. A third set of operated fish was not injected.

Repeated injections of TTX for 36 days during regeneration of the optic nerve do not cause a reduction in ganglion cell number (Edwards and Grafstein, 1983). To test for effects of longer TTX blocks, we injected three normal fish in one eye for 6 months. The retinas from both the control and TTX-injected eyes were sectioned and stained with cresyl violet. The TTX-treated retinas were still very normal in appearance, with only a slight proliferation of glial cells near the vitreal margin. Counts of more than 6000 ganglion cells from 20 sections from the eyes of these three fish showed that the blocked eyes still had approximately $90 \%$ as many ganglion cells as the opposite uninjected eyes of the same fish. Since this period of block is more than twice as long as the block used here, it appears unlikely that the bilateral TTX injections caused significant loss of ganglion cells. This conclusion is also suggested by the field potentials recorded after the block was discontinued (see "Electrophysiology," under "Results").

Anatomical techniques. A $30 \%$ solution of HRP (Sigma, type VI) in $2 \%$ dimethylsulfoxide was used to label the projection from the normal eye which is contralateral to the remaining tectum. Two different HRP protocols were used. In some fish, the nerve was crushed and injected with $1 \mu \mathrm{l}$ of the HRP solution. In other fish, $10 \mu \mathrm{l}$ of the HRP solution were injected into the vitreous of the eye. At the same time that the normal projection was labeled with HRP, the regenerating projection was labeled radioautographically by injection of $25 \mu \mathrm{l}$ of $\left[\mathrm{L}-{ }^{3} \mathrm{H}\right]$ proline $(1 \mu \mathrm{Ci} / \mu \mathrm{l}$; specific activity, $27 \mathrm{Ci} / \mathrm{mmol}$; New England Nuclear Corp., Boston, MA) into the eye ipsilateral to the remaining tectum.

Two days after the projections were labeled, the fish were anesthetized with TMS and perfused through the heart with goldfish Ringers solution, followed by $2 \%$ glutaraldehyde in Ringers. The brains were immediately removed, fixed in $2 \%$ glutaraldehyde for $6 \mathrm{hr}$, and then transferred to $30 \%$ sucrose solution overnight. Eyes from some of the fish were processed similarly, after the iris, cornea, and lens were first removed. Transverse serial frozen sections were cut at $24 \mu \mathrm{m}$ on a cryostat. Alternate sections of the tecta were saved on separate sets of slides.

One set of tectal slides was used for the histochemical demonstration of HRP. For some animals the sections were reacted with diaminobenzidine (DAB) to produce a brown reaction product (Luiten, 1975) and were counterstained with cresyl violet. For others, the sections were reacted with tetramethylbenzidine (TMB; Mesulan, 1978) to produce a blue reaction product and were not counterstained. The TMB reaction was used for all fish injected intraocularly. The DAB reaction was used for most of the fish injected into the optic nerve.

The second set of sections was processed for radioautography as described previously (Schmidt, 1978), with an exposure time of 1 month, and then counterstained with neutral red.

Tectal reconstruction and determination of transition index. Slides of double-labeled (HRP in normal projection, $\left[{ }^{3} \mathrm{H}\right]$ proline in regenerating projection) tecta were given neutral codes and pooled to prevent knowledge of their identity during analysis. Every other section of both the HRP and the radioautographic sets was drawn (camera lucida) and coded. The density of labeled fibers and terminals in each region of stratum griseum superficiale was assigned a number from 1 to 4 , with 1 being an area of very low density of retinal ganglion cell fibers, and 4 being an area of high density, as in a normal projection. Each area that was assigned a separate number was at least $200 \mu \mathrm{m}$ across. In HRP-DAB-reacted sections, an effort was made to distinguish between less label due to fewer labeled structures and that due to lighter label in the same density of structures. In neither the HRP-TMB- nor the $\left[{ }^{3} \mathrm{H}\right]$ proline-labeled sections could terminals be distinguished from fibers.

Two forms of transition index (TI) were computed to quantify the degree of patchiness. In the first method, the numerical values of the transitions in density between patches were added together. Thus, single transitions (4 to 3,3 to 2 , or 2 to 1 ) counted one, double transitions ( 4 to 2 or 3 to 1 ) counted two, and triple transitions ( 4 to 1) counted three. These values were summed over the whole tectum. To control for tectal area, the sum of the transitions was divided by the sum of the lengths of the sections (which is proportional to area) to determine the TI for that projection. In the second method, the numerical value of each transition was squared. Thus single transitions still counted one, but double transitions counted four, and triple transitions counted nine. The squaring was used so that the index would reflect the differences between gradual variations (e.g., 4-3-2-1-2-3-4, etc.) and abrupt patches (4-1-4-1, etc.), which the first method did not discriminate.

In several cases, the reconstructions of patches were checked using 
a videocamera (Hamamatsu Camera Ltd., Tokyo, Japan) mounted on a Zeiss Axiomat. The Hamamatsu camera is linear and has a threshold setting control so that patches can be defined densitometrically in the image on the video monitor (Fig. 4)

Electrophysiology. Each fish was prepared surgically as described previously (Schmidt, 1979; Schmidt and Freeman, 1980). Both optic nerves were exposed and stimulated with suction electrodes. The stimulating voltage for each nerve was selected to be 2 -fold greater than that which produced maximal evoked responses in the tectum. Responses were recorded with a Ringers-filled glass pipette and referenced to a silver chloride ground wire behind the cerebellum. The preamplifier produced a $1-\mathrm{mV}, 2-\mathrm{msec}$ square pulse just before the onset of the stimulus pulse, as a standard of comparison for the absolute amplitude of the response. Records were digitized, averaged, and stored on an LSI 11/03 microcomputer. At each tectal site, recordings were taken at 50$\mu \mathrm{m}$ depth intervals, from a depth of $500 \mu \mathrm{m}$ to the surface. The second differences of the field potentials with depth were computed to provide a representation of the sources and sinks of synaptic current (Freeman and Stone, 1969; Schmidt, 1979).

Lesions marking tectal recording sites. Recording sites were located on an exact scale drawing of the tectal surface which included the vasculature as landmarks. At the end of the recording session, the saline recording pipette was replaced with a low resistance Wood's metal-filled pipette. Recording sites were exactly relocated and the electrode was lowered to a depth of approximately $300 \mu \mathrm{m}$. Lesions were made by passing 15 to $20 \mu \mathrm{A}$ of $\mathrm{DC}$ current through the metal electrode for 1 to $2 \mathrm{sec}$.

The lesioned tecta were sectioned and processed as previously described for radioautography and HRP. Both projections were reconstructed, and the lesions placed at tectal recording sites were located in individual sections and marked on the reconstructions. The density of innervation at each lesion site was given the value assigned previously to the patch above it. The reconstructions were compared to the scale drawings of the tectal surface made at the time of recording to verify that the positions of the identified lesions were correct.

\section{Results}

\section{Anatomical studies}

Time course of segregation in control fish. Following removal of one tectal lobe in goldfish, the displaced optic fibers regenerated to the remaining tectum. As reported previously (Easter et al., 1978; Lo and Levine, 1980), they generally regenerated contralaterally to the site of the missing tectum, then entered the postoptic commissures (horizontal, minor, and transverse) to emerge at the rostral end of the ipsilateral tectum. In 11 fish, the regenerating ipsilateral projections (RIPs) were labeled from 47 to 93 days postoperatively to determine the time course of the formation of ocular dominance patches. In several cases the normal contralateral projections (NCPs) were also labeled. Before 50 days (two cases), the RIP overlapped the NCP, and the two were completely intermingled. By 50 to 60 days (three cases), the projections began to form ocular dominance patches. By 60 to 70 days (four cases), the patches were pronounced and could be demonstrated by labeling either projection. The two fish at 92 and 93 days also had pronounced patches. This time course is consistent with previous reports of fish kept at $30^{\circ} \mathrm{C}$ (Springer and Cohen, 1981; Meyer, 1982).

Effect of binocular block of activity upon segregation. Since patches were well defined in both projections by 75 days, this time point was selected to test whether a binocular block of activity prevented patch formation. Specifically, the projections of fish injected bilaterally with TTX from 18 to 75 days were compared with those receiving injections of the vehicle solution (citrate-Ringers) on the same schedule. In the seven citrateinjected control fish, the projections had formed well defined ocular dominance patches as in the uninjected controls. Figure 1 shows such patches from both the NCP (HRP labeled) and RIP ( $\left[{ }^{3} \mathrm{H}\right]$ proline labeled) of two citrate-injected control fish. The two cases, one with better defined patches than the other, illustrate the range of results seen in this group. In 10 fish having both eyes blocked from 18 to 75 days after surgery, both the RIP and NCP were much less patchy than in the corresponding citrate controls. Figure 2 shows the labeled NCP (HRP) and RIP ( $\left[{ }^{3} \mathrm{H}\right]$ proline) from two of these TTX fish, both showing little or no evidence of patchiness. These may be compared with those of Figure 1 to show the qualitative difference between the TTX-blocked and control groups. Examples of full reconstructions of the projections from a citrate- and a TTX-injected fish are shown in Figure 3.

TIs were calculated for all single tectal fish to quantify the amount of patchiness in each projection. Those listed in Table I were calculated by the second method, squaring the transition values, but all of the same conclusions were reached if the first method was used instead. For comparison, the TIs of six normal projections from normal fish were calculated. These averaged $0.08(0.04 \mathrm{SD})$ and were much smaller than those of single tectal fish. Citrate injections alone had a slight effect upon patch formation, as can be seen by comparing the TIs from citrate-injected fish $(n=10)$ with those of uninjected controls $(n=3)$. The quantitative reduction was significant $(p<0.05)$ in spite of the fact that all of the citrate-injected fish showed well developed patches (Fig. 1). The TIs calculated for TTX fish were generally lower than those for the citrate fish (Table I). Two statistical methods were used on these data. First, the Student's $t$ test (two-tailed) was employed to test for significant differences between the two groups. For the reconstructed NCPs, the difference between the citrate and TTX groups was not significant $(p<0.10)$, in spite of the great difference in the means. This was probably due to the high standard error in the citrate group. There was a highly significant difference, however, between the citrate and TTX groups in the reconstructed RIPs $(p<0.02)$, and when the values for the RIPs and NCPs were averaged together in each fish, the averages were also significantly different $(p<0.02)$. Second, we used the nonparametric Mann-Whitney $U$ test, because in some cases we noted a slight skewing in the distribution of TIs, especially for the control group. The same or greater significance levels were noted and, in addition, the differences in the NCP between the two groups were also significant $(p<0.05)$. Likewise, when these same two tests were applied to the TIs computed without squaring, all significant differences noted above were upheld. Thus, the significance of the findings does not depend upon either the method of computing the TI or the statistical best employed.

Binocular TTX block, beginning at 18 days, may have slowed rather than stopped the segregation of optic afferents. To test this, we allowed additional time for segregation in the absence of activity by extending the block to 93 to 98 days (five fish). Controls (three fish) were administered citrate injections in both eyes. There was still a significant difference between blocked and control fish at 93 to 98 days, both for the RIP ( $p$ $<0.05$ ) and for the average of the NCPs and RIPs $(p<0.05)$, as is shown in Table I, Group IIB. The TIs for the TTX fish were slightly higher than for the 73- to 78-day fish, but this difference was not statistically significant. When these additional fish were included with the 75-day group, there were significant differences between the TTX and citrate groups for the NCP $(p<0.05)$, RIP $(p<0.002)$, and the average $(p<$ 0.002 ) using both the $t$ test and the $U$ test.

Patches in long-term fish. Sixteen uninjected control fish were allowed to survive for 8.5 months after surgery to see whether there were any further changes or refinements in the ocular dominance patches. Figure 4 shows representative sections through both the NCP and the RIP for one of these fish. In general, patches were no more abundant but were generally somewhat more sharply defined. Full reconstructions of both projections from two of these fish are shown in Figure 5. When the TIs were calculated from the reconstructions of those projections, they showed an increased level of patchiness (Table 

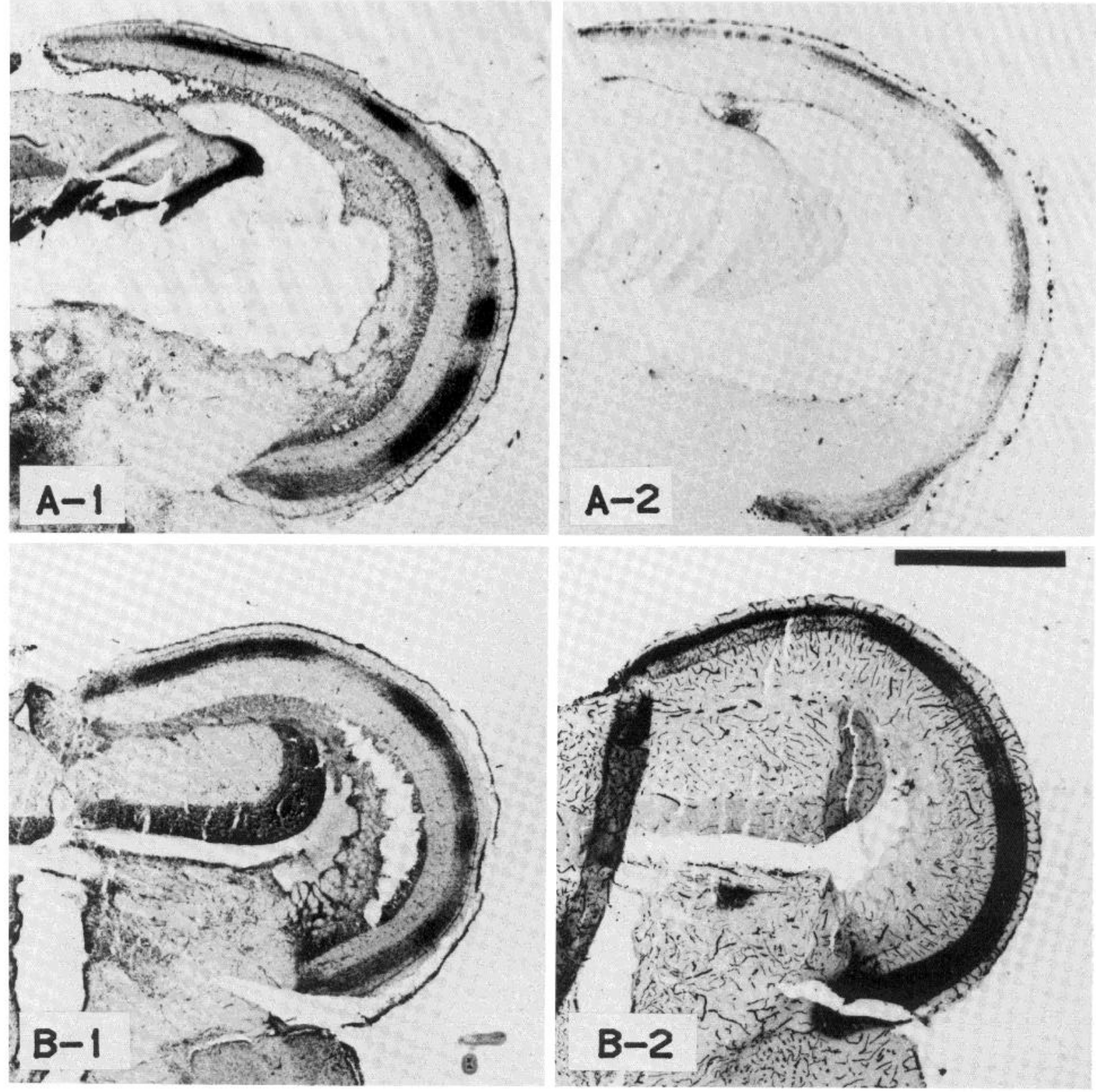

Figure 1. Tectal cross-sections from citrate-injected control fish demonstrating segregation of the projections into complementary ocular dominance patches. Dorsal is upward and lateral is to the right in all photomicrographs. Top, Adjacent sections from a citrate-injected fish at 77 days. A-1: RIP radioautographically labeled and counterstained with neutral red; $A-2$ : NCP labeled with HRP-TMB and counterstained with cresyl violet. Bottom, Adjacent sections from a second citrate fish at 74 days. $B-1$ : RIP radioautographically labeled and counterstained with neutral red; $B-2$ : NCP labeled with HRP-DAB, but with no counterstain. Many fibers of passage can be seen in the lighter areas between dense patches in this projection. The heavy superficial band seen dorsomedially is in stratum opticum, not in the terminal zone which is just below. Calibration bar: $1 \mathrm{~mm}$.

I) relative to the fish at 75 to 95 days. Differences between the groups were significant for the RIP and the average values ( $p$ $<0.002)$, but not for the NCP ( $p<0.10, U$ test or $t$ test). Thus, it would appear that there is some ongoing refinement of the patches for several months after their emergence.

Variability not attributable to tectal remnants. In all groups of fish there was considerable variability in the TIs. In most operated fish, a small remnant of the excised tectum remained intact. The average was $7.2 \%$ and the maximum was $25 \%$. We looked for a correlation between the TI and the percentage of tectal remnant to determine whether this was a cause of the variability in the TIs. If the remnants were to cause a reduction in patchiness, then a strong negative correlation would be expected between the two. To test for such an effect within each group of fish, the average TI for each fish was regressed against the percentage of tectal remnant for that fish. The regression coefficients were small, and as many were positive as negative. When they were combined statistically using the method of Winer $(1962)$, no correlation was found $(p<0.95)$. Therefore, the variability in the TIs was not due to the presence or size of the tectal remnants.

Complementary organization of the patches in the two projections. Consistent differences in the degree of patchiness of the NCP and RIP were seen in all control fish. Generally, the regenerated projection was patchier than the resident contralateral projection (Table I). Also, the NCP tended to occupy a greater percentage of the tectal area than the RIP. The reconstructions in Figures 3 and 5 illustrate the relative areas occu- 

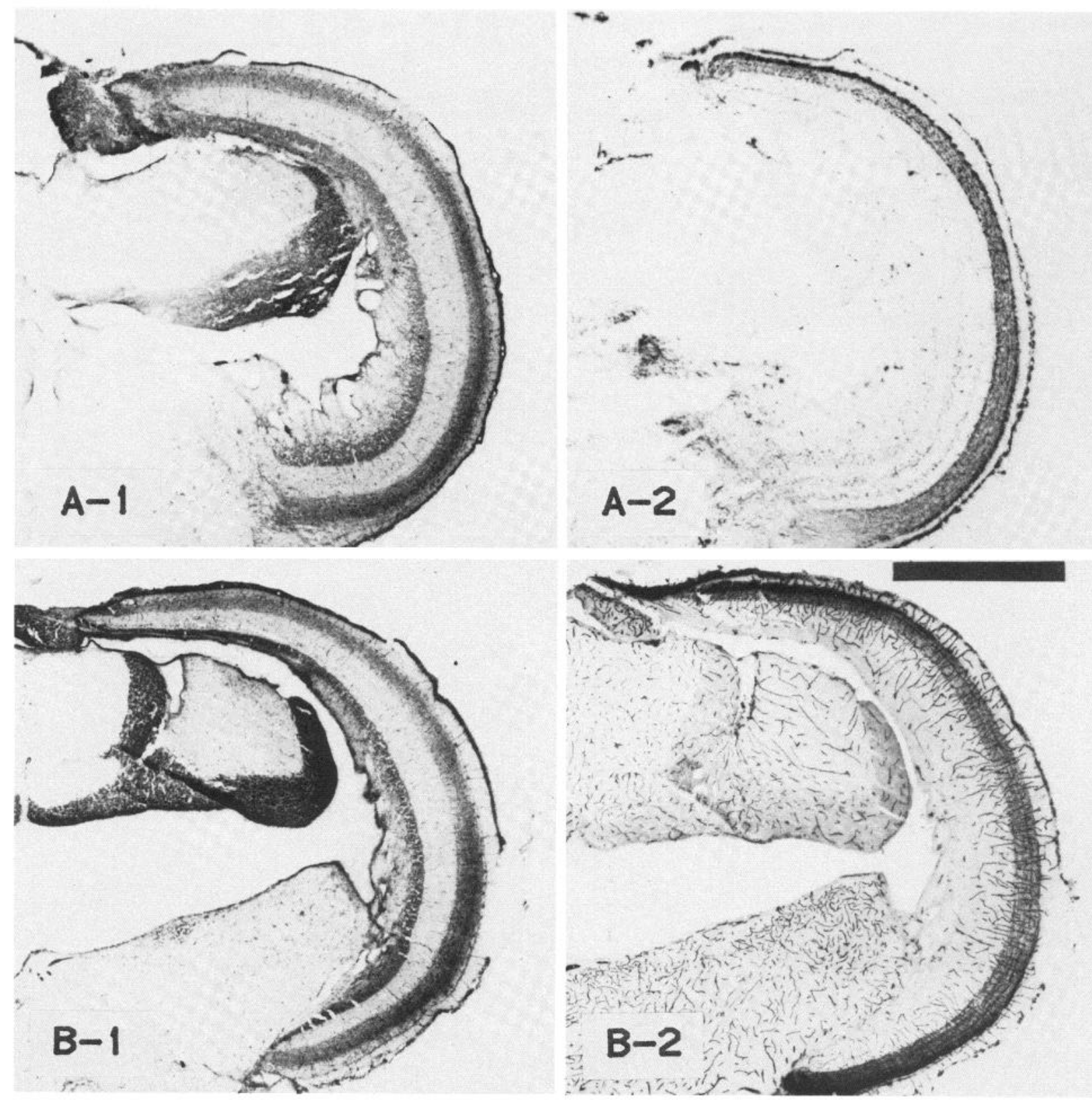

Figure 2. Tectal cross-section from TTX-injected fish showing marked decrease in patchiness in both projections. Top, Adjacent sections from a TTX-injected fish at 74 days. $A-1$ : RIP labeled with radioautography and counterstained with neutral red; $A-2$ : NCP labeled with HRP-TMB, and no counterstain. Bottom, Projections from a second TTX-injected fish also at 74 days. B-1: RIP radioautographically labeled and counterstained with neutral red; $B-2$ : NCP labeled with HRP-DAB, and no counterstain. Calibration bar: $1 \mathrm{~mm}$.

pied by the projections and the degree of overlap between them. These observations led us to make a quantitative assessment of the degree to which a high density in one projection corresponded to a low density in the other projection. To do this we took systematic readings of the densities from adjacent sections processed for radioautography and HRP at corresponding fixed points. Using the reconstructions, observations were made at $1-\mathrm{cm}$ intervals across each drawing. This corresponded to a sampling interval of just over $200 \mu \mathrm{m}$ in the original tissue and yielded several hundred pairs of observations in each fish. The data for three groups of fish are plotted in Figure 6. For TTXblocked fish, there was no significant tendency for the HRP density to be lower where the radioautographic density was greater. In fact, the plot is rather flat, consistent with the conclusion that the two projections are thoroughly intermingled. For the 75-day control fish and the uninjected controls at
8.5 months, there was a significant tendency for densely labeled areas in the radioautographs to have lower densities of HRP labeling at those sites. This tendency was slightly more pronounced in the 8.5-month fish. However, the plots of densities were far from complementary. Thus, in areas where the radioautographic density was 1 , the HRP density averaged approximately 3.5 ; but in areas where the radioautographic density was 4 , the HRP density, although lower, was still approximately 2.8 to 2.9 . In the very best case, that shown in Figure 4, the HRP density averaged 1.99 in regions where the radioautographic density was 4 .

There are several reasons for this less than complementary relationship, some of which are evident in the reconstructions. First, the high density areas in one projection were almost always slightly larger than the corresponding holes in the other projection, leading to a degree of overlap. This conclusion from 

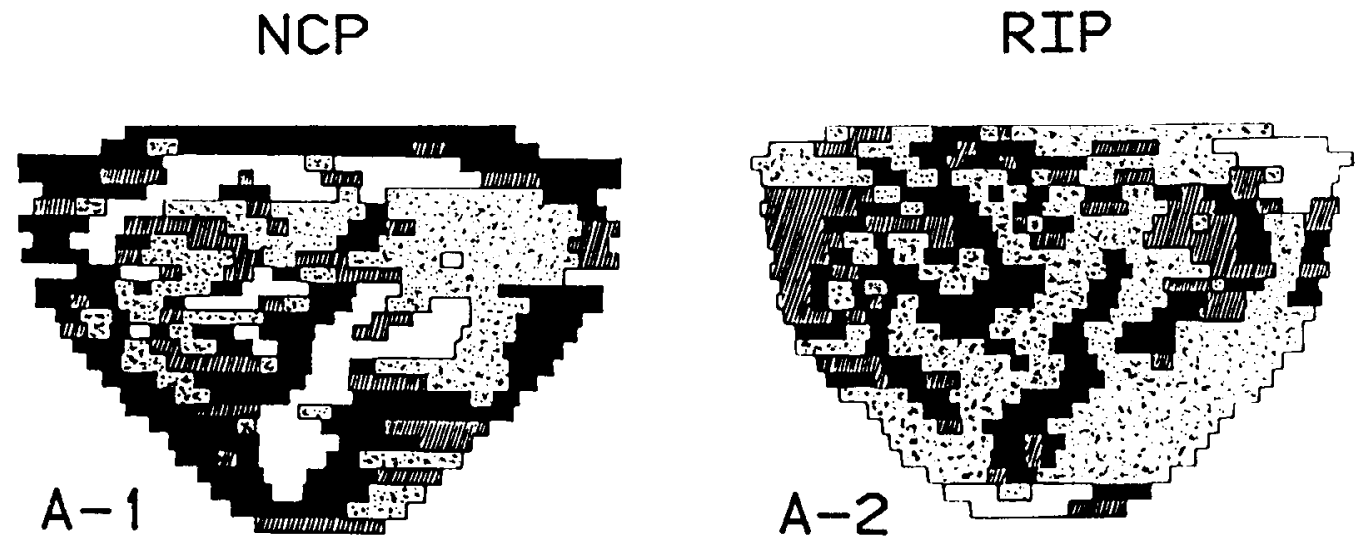

Figure 3. Tectal reconstructions from a citrate-injected (top) and a TTX-injected (bottom) fish. On the left are the NCPs and on the right the RIPs. In each section, the density of label in each patch was assigned a number from 1 to 4 , with 1 being an area of very low density and 4 being an area of high density, as in a normal projection. In these drawings, white areas $=1$, stippled areas $=$ 2 , striped areas $=3$, and black areas $=4$. A comparison of the upper set of reconstructions with the lower set demonstrates the reduction in segregation in TTX-
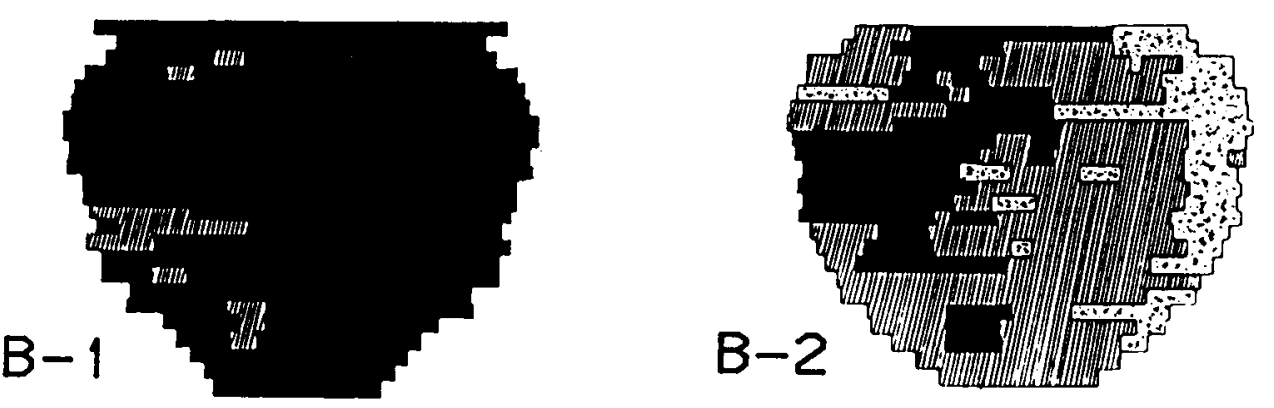
injected fish relative to the citrate-injected controls. The TIs for the control projections were 1.77 $(N C P)$ and $1.64(R I P)$; for the TTX fish, they were $0.06(N C P)$ and $0.28(R I P)$. Rostral is at the top; medial is to the right.

TABLE I

Effect of TTX on patch formation: MeanTIs

\begin{tabular}{lllll}
\hline $\begin{array}{c}\text { Group (days } \\
\text { postoperative) }\end{array}$ & \multicolumn{1}{c}{ Injections } & NCP (SEM; $N$ ) & RIP (SEM; $N$ ) & $\begin{array}{c}\text { Average of NCP } \\
\text { and RIP }\end{array}$ \\
\hline I & None & & $2.30(0.54 ; 3)^{\alpha}$ & \\
(70-93 days) & Bilateral citrate & $1.12(0.36 ; 7)$ & $1.18(0.21 ; 6)$ & $1.13(0.25 ; 7)$ \\
IIA & Bilateral TTX & $0.36(0.13 ; 10)$ & $0.45(0.14 ; 10)^{b}$ & $0.42(0.12 ; 10)^{b}$ \\
$(73-78$ days) & Bilateral citrate & $1.02(0.58 ; 3)$ & $1.12(0.18 ; 3)$ & $1.07(0.11 ; 3)$ \\
IIB & Bilateral TTX & $0.57(0.13 ; 5)$ & $0.52(0.13 ; 5)^{c}$ & $0.55(0.11 ; 5)^{c}$ \\
(93-98 days) & Bilateral citrate & $1.06(0.27 ; 10)$ & $1.33(0.18 ; 9)$ & $1.20(0.19 ; 10)$ \\
IIC & Bilateral TTX & $0.43(0.10 ; 15)^{c}$ & $0.48(0.10 ; 15)^{d}$ & $0.46(0.10 ; 15)^{d}$ \\
(73-98 days) & None & $1.60(0.25 ; 14)$ & $3.20(0.15 ; 13)^{d}$ & $2.31(0.18 ; 16)^{d}$ \\
III & & & & \\
(255 days) & & & & \\
\hline
\end{tabular}

${ }^{a}$ All injected fish have significantly lower TIs in RIP than do uninjected fish.

${ }^{b}$ Significantly different from citrate control, $p<0.02$.

${ }^{c}$ Significantly different from citrate control, two-tailed $t$ test, $p<0.05$.

${ }^{d}$ Significantly different from citrate control, $p<0.002$.

the reconstructions was verified using video microscopy as shown in Figure 4 . This pair of projections, as judged by the human eye, was both sharply segregated and extremely complementary. Nevertheless, when the patches were defined by densitometric cutoff and quantitative measurements were taken, the dense bands of each projection were always larger than the holes in the other. When the dense radioautographic bands were compared to the HRP holes, they averaged $16 \%$ bigger (range, 7 to 25\%). Likewise, the dense HRP bands were, on average, $18 \%$ bigger than the holes in the radioautographic bands (range, 12 to $33 \%$ ). This effect is real even in the best segregated cases and contributes to the lack of total complementarity seen in Figure $6 C$. Second, in a few cases such as that reconstructed in Figure $5 B$, the HRP-labeled projection was not patchy over its entire extent, even though the other projection had sharply defined patches in all areas. This occurred in at least one-fourth of the cases for no apparent reason. Third, some degree of overlap is to be expected because of labeled axons of passage in the low density areas between high density patches. This causes overlap to be much more prominent rostrally where the axons enter (Figs. $3 A$ and 5). Finally, there may be a small methodological component, due to problems in locating the exactly corresponding points in the adjacent sections. Differential shrinkage of the sections processed for HRP reaction and radioautography was less than $4 \%$ and, therefore, did not introduce any substantial error. Observations were checked by a second observer, yielding good agreement. The measurements therefore do not reflect any systematic error, but rather the limited degree of complementarity in the projections. 

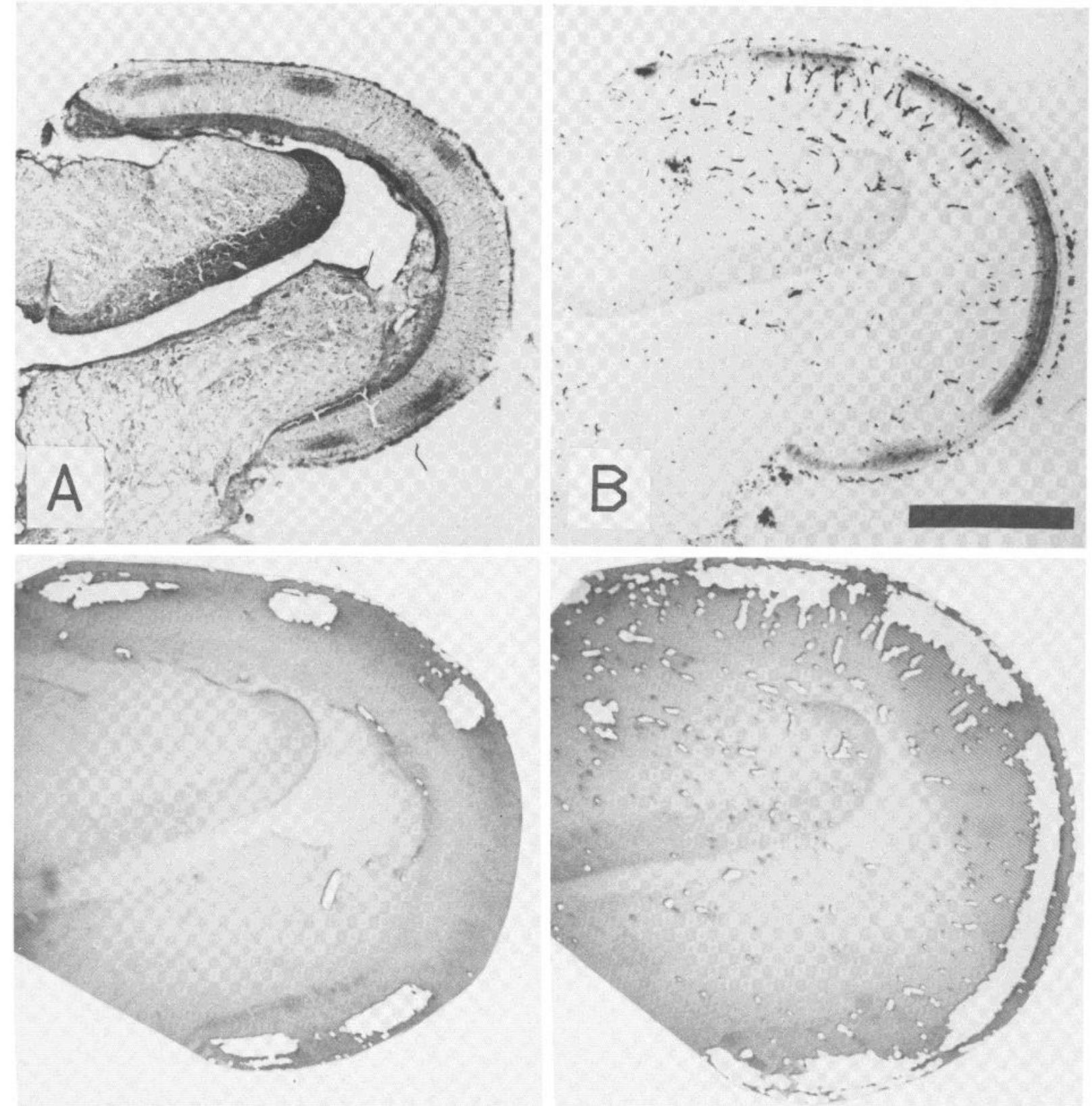

Figure 4. Tectal cross-sections from an 8.5-month control fish demonstrating sharp ocular dominance patches. $A$ and $B$, Adjacent sections from the RIP (radioautography, neutral red stain) and NCP (HRP-TMB, no counterstain), respectively. Below are videomicroscopic images of the same sections photographed from the television monitor. To quantify the lengths of the patches, a threshold level has been set in the camera such that all areas with greater density appear white. The HRP and radioautographic labeling had nearly identical ranges of densities, so that the same threshold was used for both. Note the good agreement between the threshold-defined patches and those in the reconstruction (Fig. 5 , arrows). Calibration bar: $1 \mathrm{~mm}$.

Nevertheless, there was still a significantly lower density of NCP labeling in areas with high RIP labeling. The level of significance was determined using pairwise comparisons within each fish. For example, the average HRP density where the radioautographic density was 4 was subtracted from the average HRP density where it was 1 , and then a $t$ test was used to determine whether the mean of the differences across the group was significantly different from zero. This method eliminated any variation between fish in overall labeling density. For the TTX fish, no pairwise differences were significant, even if 1's and 2's (low density areas) were combined and tested against combined 3's and 4's (high density areas). For the two control groups, this same test was significant at the 0.005 level. In addition, the HRP densities in the areas where the radioautography was rated 4 were always significantly lower in pairwise tests than the HRP densities in areas where the radioautography was rated 1,2 , or 3 . Thus, there was significant evidence of a tendency to segregate, although this segregation was not complete.

\section{Electrophysiology}

To determine whether there was an electrophysiological correlate to the anatomical ocular dominance patches, we compared the postsynaptically generated field potentials, elicited by shocking either the right or the left optic nerve, at several tectal sites. In normal unoperated fish the amplitudes of the 
NCP

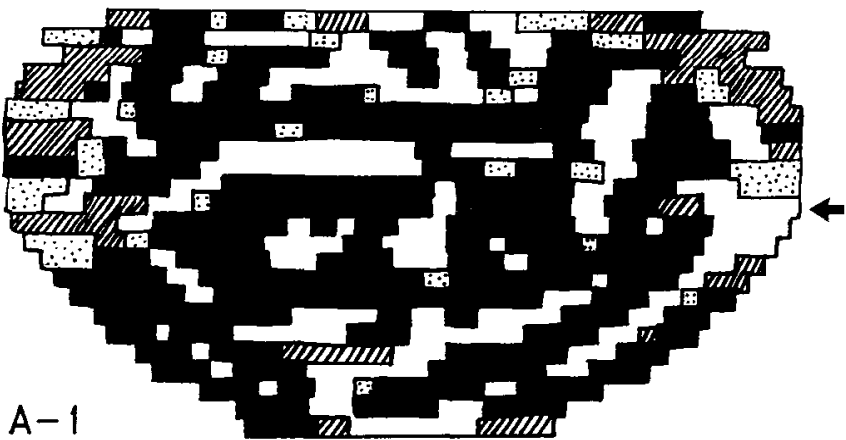

A-1

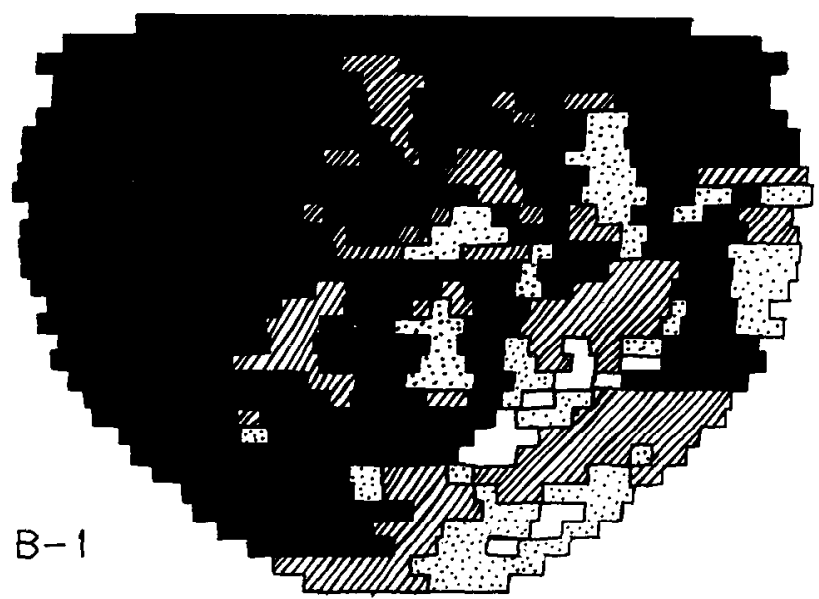

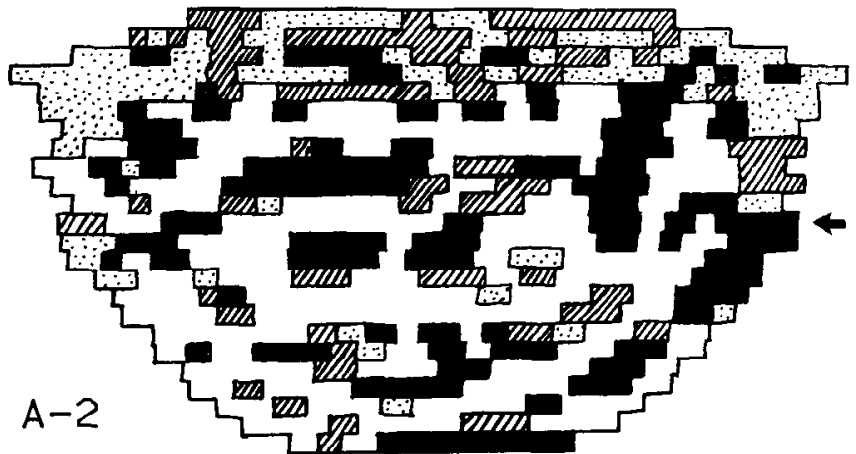

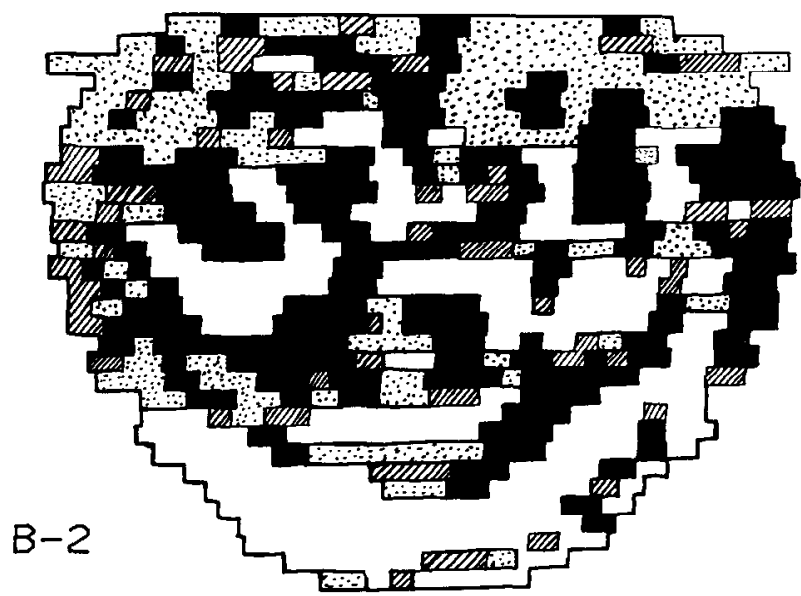

Figure 5. Tectal reconstructions from two 8.5-month control fish, showing the extremes in complementarity of the patches. $A-1$ and $A-2$, NCP and RIP projections from the same fish as the sections shown in Figure 4. Arrows indicate the reconstructions of those sections. The TIs computed for these projections were $3.87(N C P)$ and $3.39(R I P), B-1$ and $B-2$, Projections from a second 8.5-month control fish showing less complete segregation. The TIs were $0.83(N C P)$ and $2.96(R I P)$, reflecting the relative lack of patches in the NCP. Conventions are as in Figure 3.

field potentials do not vary appreciably across the tectum (J. T. Schmidt, unpublished results): We therefore searched for variations in the amplitudes of the evoked field potentials, or in the sources and sinks of synaptic currents, for each projection in single tectal fish. Such variations would be expected if the synaptic input from a projection varied across the tectum as suggested by the anatomy. $\Lambda$ t each site, recordings were made at $50-\mu \mathrm{m}$ depth intervals, beginning at a depth of $500 \mu \mathrm{m}$ and proceeding upward toward the surface. Recordings were repeated across the tectum in a mediolateral line (perpendicular to the orientation of the anatomical patches) at sites which were 0.4 to $0.8 \mathrm{~mm}$ apart. Forty-five penetrations were made in 12 uninjected 8.5-month fish, 10 penetrations were made in 4 citrate-injected fish, and 13 penetrations were made in 5 TTX-injected fish. Example recordings of field potentials from TTX fish and uninjected long-term controls are shown in Figures 7 and $8 C$. By 75 days the regenerating ipsilateral projection had established effective synaptic connections in the remaining tectum as shown by the substantial field potentials elicited by shocking the ipsilateral optic nerve. This was true for both citrate- and TTX-injected fish, indicating that activity was not needed to establish some synaptic connections even though the tectum was already occupied by the usual contra- lateral projection. The sources and sinks for both projections were distributed in the normal pattern (Schmidt, 1979). The major sinks were between 100 and $200 \mu \mathrm{m}$ in depth correspond ing to the major optic recipient lamina (superficial gray and white layer-heavy bands in the anatomy). There were also longer latency secondary sinks deeper, at approximately 300 $\mu \mathrm{m}$ (central gray layer). Thus, after the invasion of the RIP, the two projections shared the projection lamina which was previously innervated exclusively by the contralateral.

In most single tectal fish, large variations in the amplitudes of both the field potentials and the synaptic sources and sinks were found (Figs. 7 and $8 C$ ). If the complementary organization seen in the anatomy had an electrophysiological correlate, then areas with large ipsilateral responses should have small contralateral responses and vice versa. We looked for such a relationship by plotting the amplitude of the ipsilateral responses versus the contralateral responses for all three groups of fish (Fig. 9). For the uninjected 8.5-month control fish, there was a significant relationship between the amplitude of the two responses from the two eyes. As expected, penetrations with large ipsilateral responses generally had smaller contralateral responises. In nearby penetrations where the ipsilateral response was small, the contralateral response was large. This relation- 

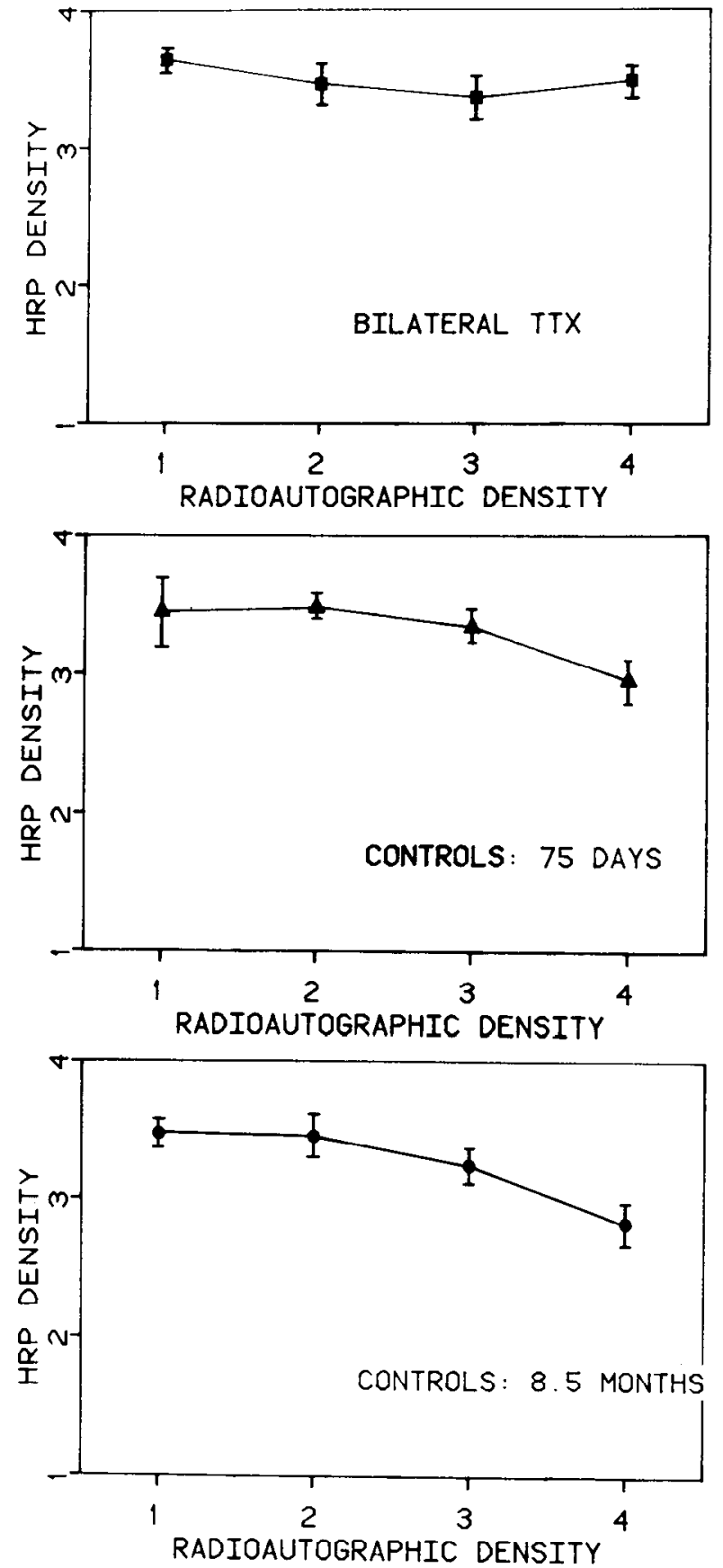

Figure 6. Plots of the density of HRP labeling of the NCP versus density of radioautographic labeling of the RIP in three groups of fish. Top, TTX-injected fish $(n=10)$ at 75 days. Middle, Citrate-injected control fish $(n=6)$ at 75 days. Bottom, Uninjected controls at 8.5 months $(n=13)$. The error bars indicate the standard errors of the mean at each point. Note the flat function for the TTX fish and negative slopes in the controls.

ship was usually more marked within each fish, but because of variability in overall responsiveness between fish, there is a lot of scatter when the points are plotted together. A regression line fitted through the points had a negative slope significantly different from zero $(p<0.005)$, confirming the reciprocal relationship. In the 75-day citrate controls, very few penetrations were found that had large ipsilateral and small contralateral responses. The same regression for the citrate-injected controls gave nearly the same negative regression slope (not plotted), but it was not significantly different from zero. The distribution of ipsilateral response size in TTX fish was virtually flat, meaning that the ipsilateral projection was almost as likely to give its largest responses where the contralateral response was large as where it was smaller. There were no sites where the ipsilateral responses were much larger than the contralateral responses. This is consistent with the lack of segregation in TTX fish seen anatomically. The data, particularly for the 8.5month controls, show electrophysiologically the same tendency for a segregation of the tectum between the two projections as seen in the anatomical results.

Differences in responses for the RIP and NCP. There were some general differences in the recordings for the RIP and NCP. In the 75-day dish, injected with either TTX $(N=5)$ or citrate $(N=4)$, the responses elicited from the RIP were nearly always smaller than those from the NCP, as shown in Figures 7 and 9 . In addition, the field potential waveforms occurred at longer latencies for the RIP than for the NCP and were longer in duration, indicating a slower nonuniform conduction velocity for the regenerated fibers. These differences are typical of regenerating fibers during the first few months (Schmidt et al., 1983) and reflect the fact that fibers are small and nonuniform in diameter, are nonmyelinated or poorly myelinated (Murray, 1982), and have not yet regained normal synaptic density at most recording sites. In the 8.5-month fish, the maximum negative deflections of the field potentials and the integrated areas of the synaptic sources and sinks were sometimes actually larger (13 of 45 cases) for the RIP than for the NCP at some recording sites. However, averaging over all of the penetrations, even in the 8.5-month fish the field potentials for the RIP were generally smaller than those of the NCP (average, 69\%). Also, in most cases, the long latencies and durations of the field potentials from the RIP could still be seen at 8.5 months. The longer latency responses tended to be associated with low amplitudes. This relationship is plotted in Figure 10. A regression analysis gave a negative slope which was significantly different from zero ( $p<0.05$, two-tailed $t$ test). These data indicate that many optic fibers in the RIP were not fully mature, even at 8.5 months after surgery, although regenerated projections normally mature within 5 to 6 months after nerve crush (Schmidt et al., 1983).

Correlation between electrophysiology and anatomy. Lesions were made at tectal recording sites in double-labeled fish and were later located anatomically in six fish. One such lesion is shown in Figure $8 A$. Figure $8, B$ and $C$, shows part of a reconstructed tectum (RIP only) containing lesion sites and the field potentials recorded at two of these sites. In order to test for a correlation between the density of innervation at the lesion site and the amplitude of the field potentials in each projection, the density of label at each recording site was compared to the amplitude of the evoked response at that site. Since treating each projection of each fish separately provided very few observations (three to five) per group to use for statistical analysis, we pooled the data for ipsilateral and contralateral projections across fish. Pooling across all fish produced significant positive regression coefficients $(p<0.005$ for the RIP and $p<0.01$ for the NCP) in spite of the differences in the absolute amplitude of responses between fish. These data are illustrated in Figure 11. Note that the regression lines for the two projections have substantially different slopes because the ipsilateral responses were generally somewhat smaller. As an alternative to pooling across fish, we pooled the projections within each fish and found a significant positive regression coefficient $(p<0.05)$ in three of the six fish. The other fish produced positive but not significant regression coefficients. When combined statistically by the method of Winer (1962), the results were significant at the 0.005 level. Thus, we found a good correspondence between the amplitude of the field 

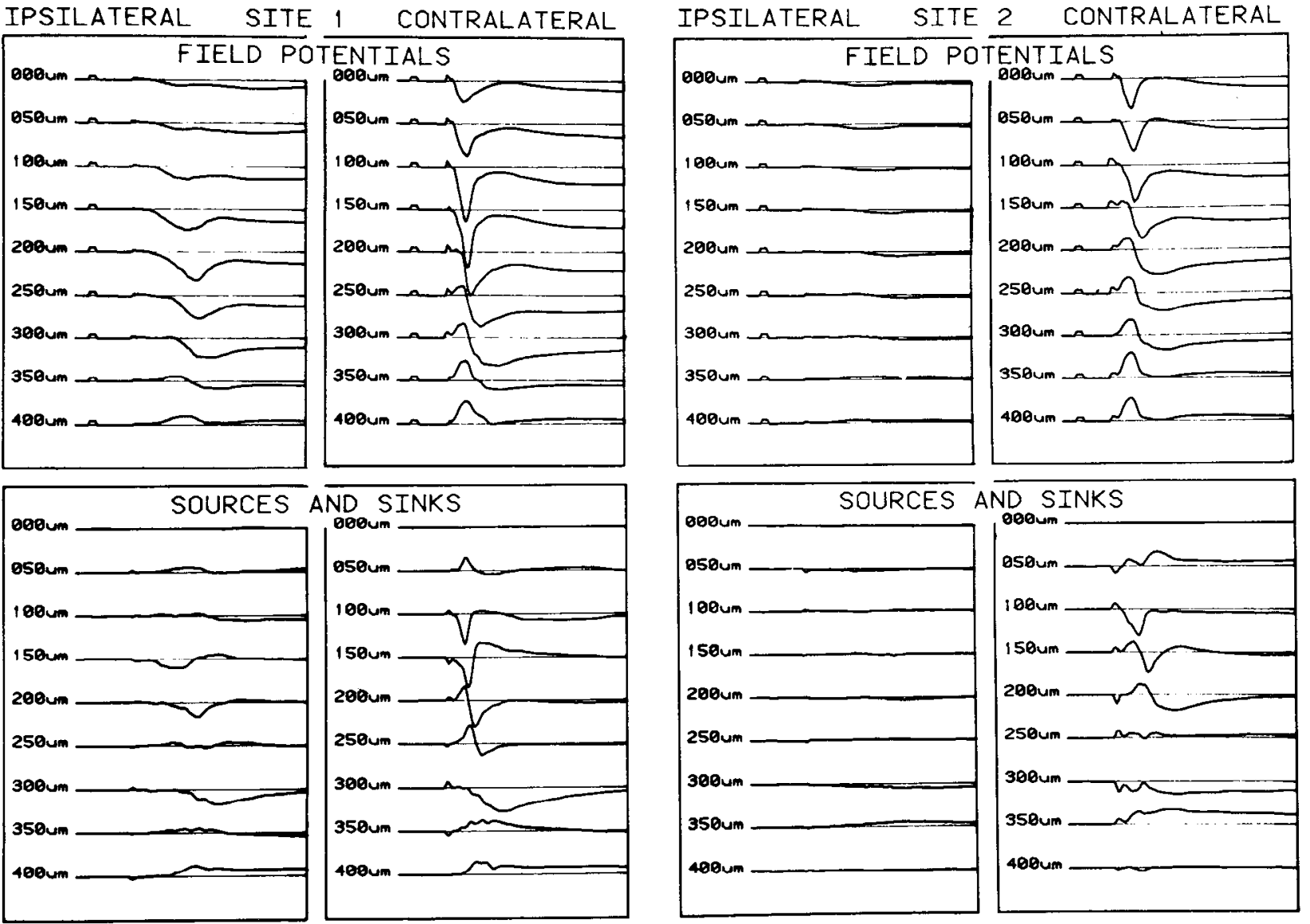

Figure 7. Field potentials evoked by optic nerve shock in fish bilaterally injected with 'TTX. At the top are the averaged field potentials for the NCP and RIP at two different recording sites. The length of each trace is $80 \mathrm{msec}$, and the calibration pulse is $1 \mathrm{mV}, 2 \mathrm{msec}$. The lower sets show the corresponding sources and sinks of synaptic current. The depth of each recording is shown at the left of each trace. Negative is down in the field potentials, and sinks are downward deflections in the lower traces.

potentials at each point and the density of innervation assessed by anatomical labeling.

Taken together, the variations in amplitude of the evoked responses within the NCP and RIP across the tectum, the complementary nature of these variations, and the correlation between label density and synaptic strength at lesions sites all furnish strong evidence that an electrophysiological correlate exists for the anatomical ocular dominance patches.

\section{Discussion}

There are three major conclusions from this study. First, from 50 to 70 days postsurgery the RIPs and NCPs segregate into ocular dominance patches. Second, a binocular block of activity from 18 to 95 days prevents segregation of the RIP and NCP. Third, there is an electrophysiological correlate of the anatomical ocular dominance patches. We will first discuss our electrophysiological results. Then we will examine the possible mechanisms for segregation and how activity may be involved.

\section{Electrophysiological correlate of the ocular dominance patches}

This study demonstrated an electrophysiological correlate to ocular dominance patches in several ways. First, the amplitude of the field potentials from each eye varied across the tectum in a manner similar to that of the anatomical labeling. Second, the amplitudes of the field potentials from the two eyes were roughly complementary; that is, where the ipsilateral response was large, the contralateral response tended to be much smaller than usual, and vice versa. Third, we used lesions to correlate the amplitude of the evoked field potentials with the density of innervation from that eye. There was a statistically significant relationship between fiber density and synaptic strength, in spite of several factors which may have lessened the correlation. First, our recording method involved relocating the recording sites with a metal electrode for making lesions. This was necessary because lesioning while recording at each site might well have interrupted passing fibers en route to the next recording site. Our relocation of the recording sites could have been in error by as much as $50 \mu \mathrm{m}$. Second, we found it necessary to pool data from each projection across all six fish due to the small number of recording sites per fish. Although the maximum responses sometimes varied by a factor of 2 between fish, there was a highly significant regression between label density and amplitude of field potentials (Fig. 11). Previous studies looked at presynaptic activity (Levine and Jacobson, 1975; Schmidt, 1978; Law and Constantine-Paton, 1981) and found that fibers from the two eyes could be recorded at almost all tectal sites. Occasionally in the frog (Law and Constantine-Paton, 1981) and goldfish (Schmidt, 1978), the unit responses from one eye were larger and predominated. The use of field potentials in this study is particularly appropriate since they are postsynaptically elicited and therefore reflect the density of optic synapses rather than the presence of fibers or branches of terminal arborizations merely passing through the area. With this technique, a strong correlation was found between the electrophysiology and anatomy.

The recording of field potentials was not as good a method as anatomical labeling for assessing the degree of segregation between the RIP and NCP, simply because so few points could be sampled in each fish. Nevertheless, the electrophysiological results demonstrated that there is a functional difference be- 


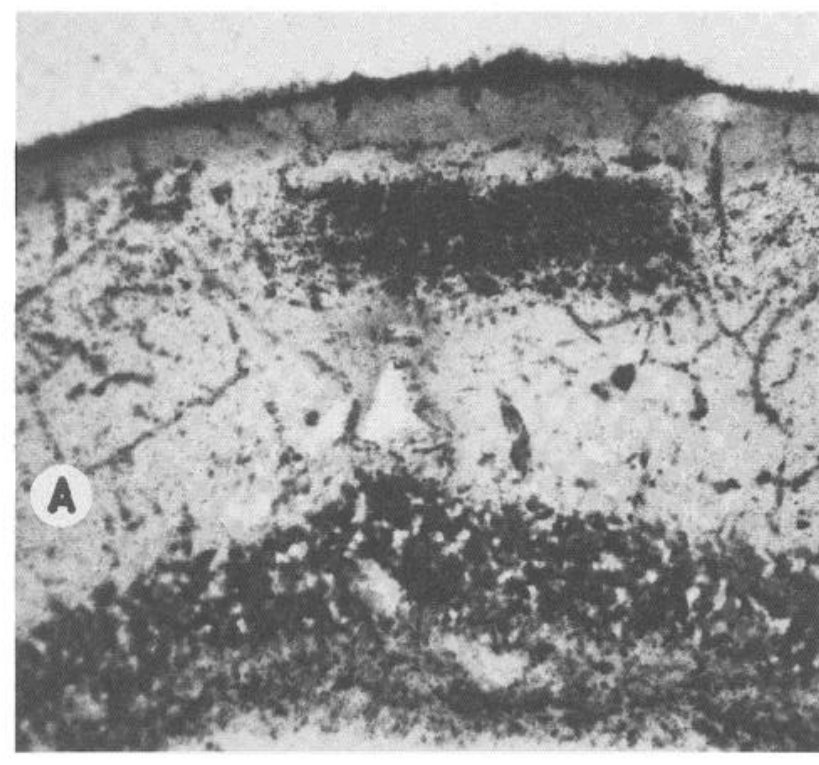

C

IPSILATERAL SITE 2 CONTRALATERAL
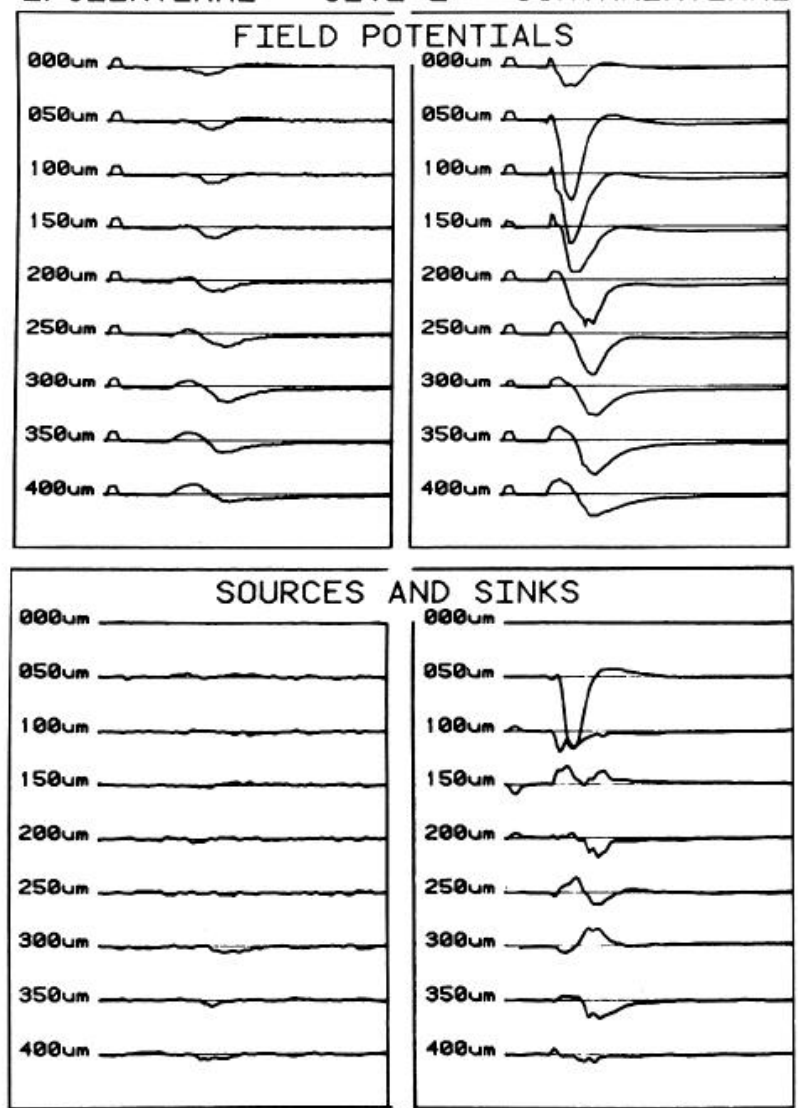

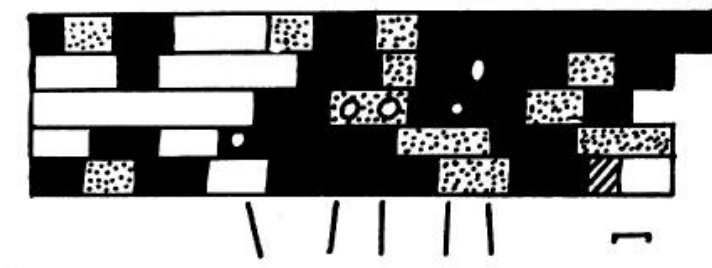

B. SITES 123345 100um
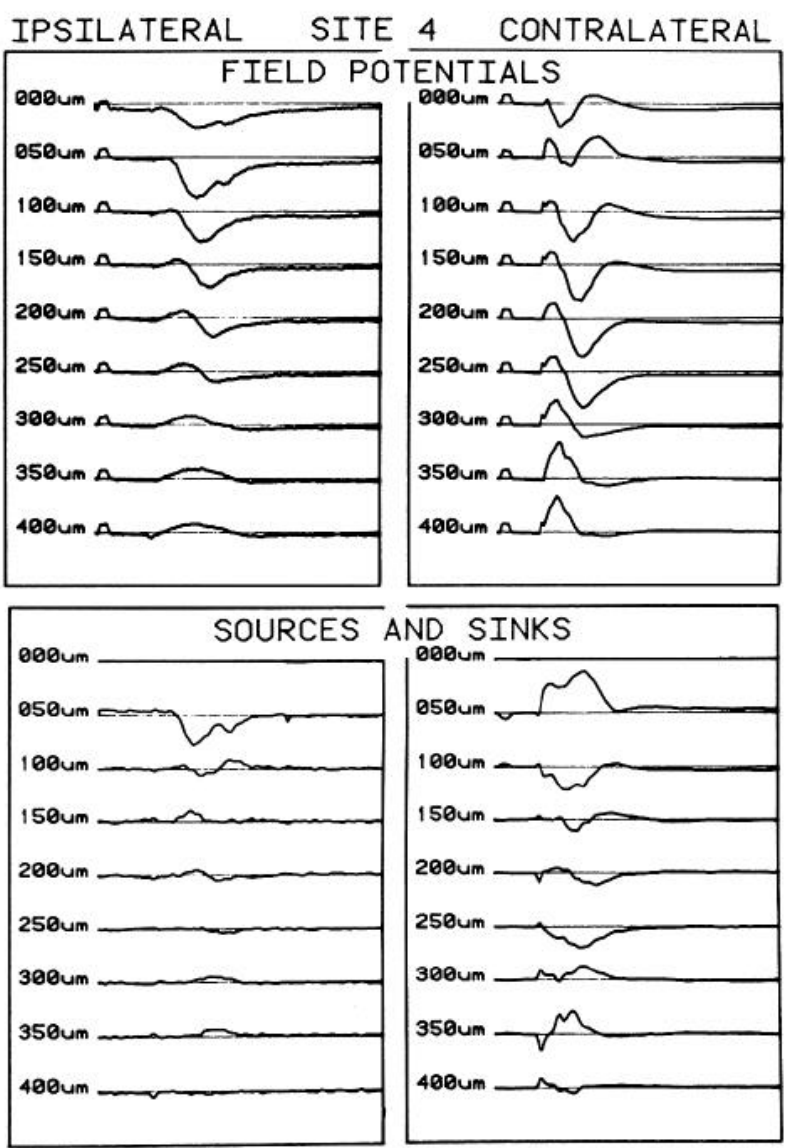

Figure 8. Correlation of anatomy and electrophysiology using lesions in a long-term fish. A, An example of a lesion placed at a tectal recording site at a depth of approximately $300 \mu \mathrm{m}$. The lesions were usually about $50 \mu \mathrm{m}$ in diameter. $B$, Location of lesions marked on part of a reconstruction of the RIP in a long-term TTX-RIP fish. The lesions are labeled 1 through 5 . $C$, Sets of field potentials for recording sites 2 and 4. In $B$ at site 2, the density of labeled fibers is low and the field potentials elicited from the RIP are small in amplitude. At site 4 in $B$, the density of labeled fibers was high and the field potentials elicited from the RIP were much larger, illustrating the general correspondence between anatomy and electrophysiology. 

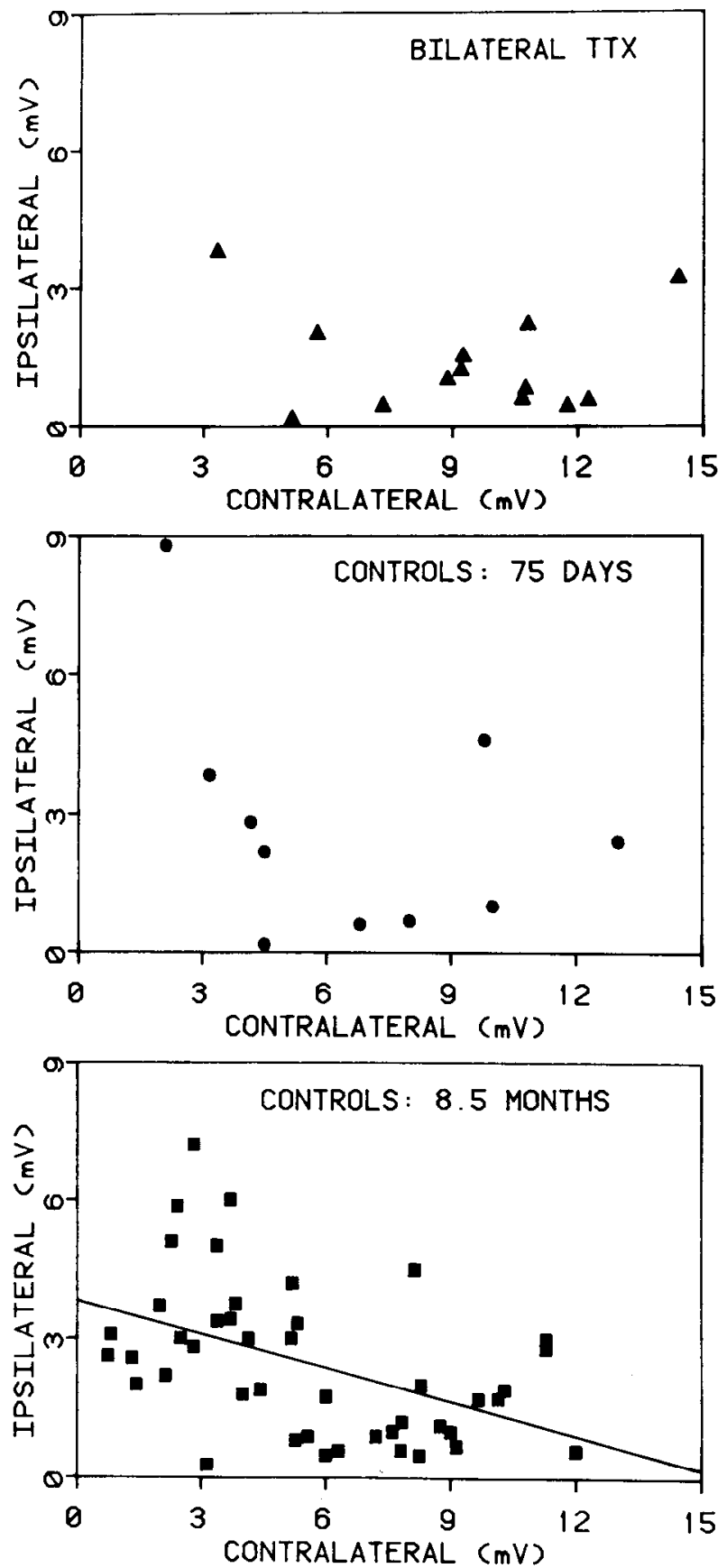

Figure 9. Plots of the relationships between the amplitudes of ipsilateral and contralateral field potentials for three groups of fish. Top, Bilateral TTX fish at 75 days. Middle, Citrate-injected controls at 75 days. Bottom, Uninjected controls at 8.5 months. Each symbol represents a single penetration. A significant regression line is drawn in the bottom plot. The data for the citrate-injected controls could be fitted with a line of similar slope, but the fit was not statistically significant. The data for the TTX fish are best fit by a flat line (not significant).

tween high and low density patches in either projection. The recordings were also very useful in demonstrating that effective synapses were made in the absence of activity. This was true for the regenerating projections (Fig. 7), which were blocked from 18 to 75 days after surgery, a time period during which synaptogenesis as well as segregation occurs. The failure of TTX to block synaptogenesis during regeneration was also noted by Schmidt et al. (1983). Since the number of postsynaptic sites probably did not increase (Murray et al., 1982), the RIP must have been able to capture some synapses from the

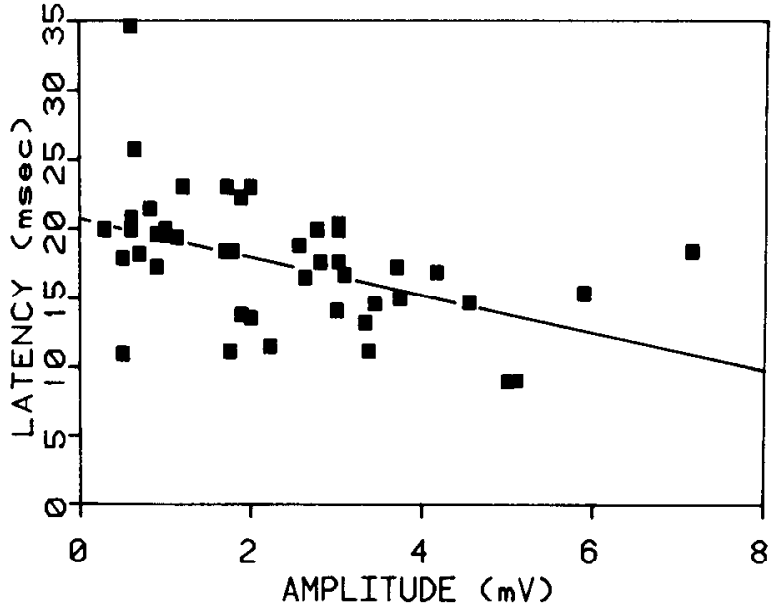

Figure 10. Plot of the relationship between the latency to peak response versus amplitude of the field potentials in the ipsilateral projections of twelve 8.5-month control fish, showing the tendency for small responses to have long latencies. Each symbol represents one penetration. The regression line has a negative slope, significantly different from zero $(p<0.01)$.

NCP, even without activity. Also, since the largest field potentials of the RIP were never equal to the largest of the NCP, either during early segregation or after patch formation at 8.5 months, there appeared to be a general difference in overall synaptic efficacy between the two projections. In fact, there appeared to be a general retardation in the maturation process after regeneration in the RIP. In a study of the re-establishment of synaptic transmission after optic nerve regeneration in goldfish, Schmidt et al. (1983) found that conduction velocity of the optic fibers had returned to nearly normal levels by 161 days post-crush. In the present study, conduction velocity in the RIP remained very much subnormal even at 255 days ( 8.5 months), as the latencies, even after correcting for the longer path traveled, were still much longer than those for controls. Furthermore, the shapes of the waveforms in many cases resembled those of newly regenerated projections, being long in latency and duration and low in amplitude. This association of low amplitude with slow conduction velocity supports the suggestion (Wolburg, 1978) that maturation of regenerated optic fibers may be linked with the establishment of effective connections.

Possible mechanisms for segregation. After the displaced projection regenerated to the remaining tectum, the two projections initially overlapped, then segregated over the next 2 months. Several mechanisms could be responsible for the segregation (Fawcett and Willshaw, 1982), including the following. (1) Fibers from the two eyes may have different markers, enabling right eye fibers to distinguish themselves from left eye fibers. These could be diffusible substances transported from the retina or fixed membrane markers which adhere to like markers better than to unlike markers. (2) The fibers from either eye may remain segregated in the optic tract and tectum resulting in their termination in separate patches. (3) Synapses may be most stable when axonal arbors from neighboring retinal ganglion cells terminate near one another because neighboring cells have similar patterns of activity.

The first mechanism, which relies on fibers recognizing specific left-right markers, is unlikely because two left eyes or two right eyes will form patches equally well even if on the same side of the head (Law and Constantine-Paton, 1981). In addition, patches are formed by compound eyes, both the surgically grafted type (Fawcett and Willshaw, 1982) and those formed spontaneously by early injury or partial removal of the eye cup (Ide et al., 1983). 

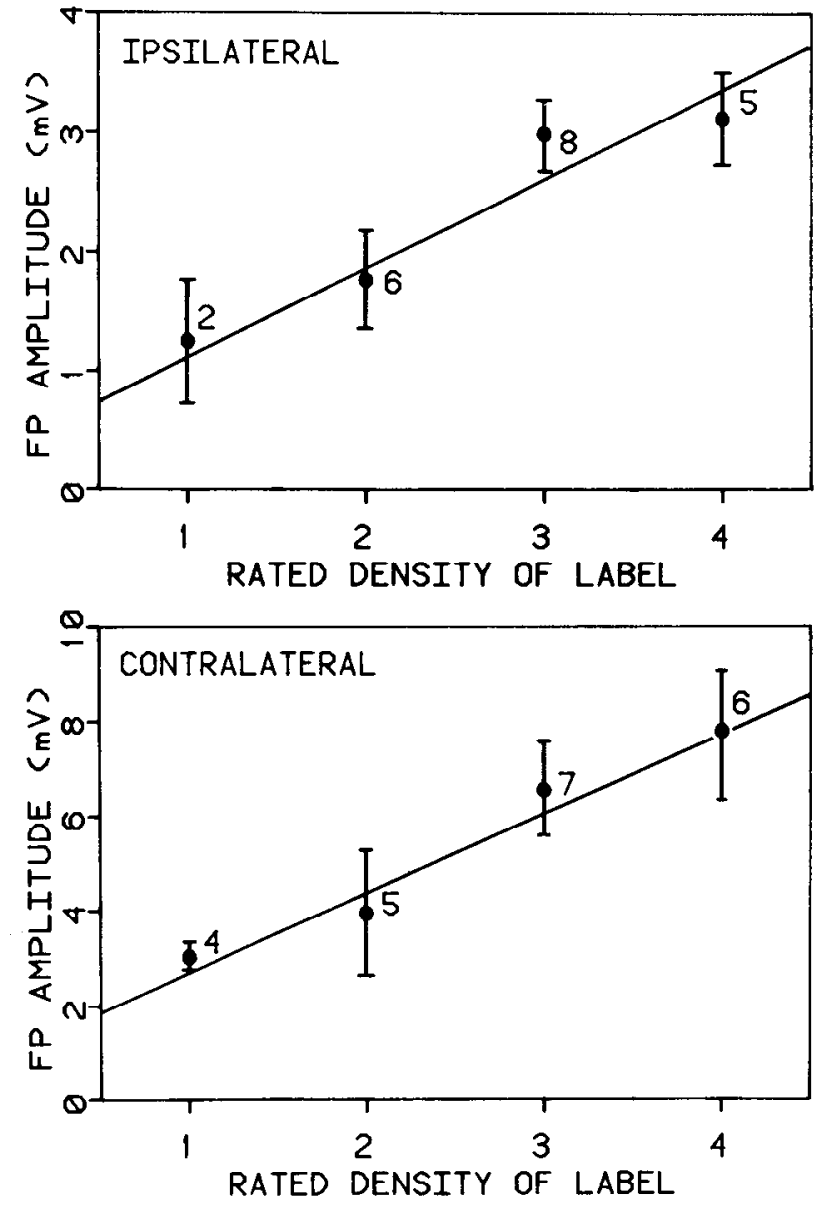

Figure 11. Correlation of field potential amplitude with density of labeling. The amplitude of the field potentials (ordinate) was regressed against the density of labeling at each lesion site (abscissa). A, Regression line for the ipsilateral projection using pooled data from six fish The slope of the regression line was significantly different from zero $(p<0.005)$. $B$, Regression line for the contralateral projection using pooled data from six fish. The slope of the regression line was significantly different from zero $(p<0.01)$. The symbols and error bars give the mean amplitudes and standard errors of the field potentials associated with each density level. The numbers give the number of lesions at each density level.

The second mechansim (fibers from the two eyes remain separate until they terminate) has also been ruled out. The optic tracts of three-eyed frogs were not separated in the diencephalon (Law and Constantine-Paton, 1981). Also in Xenopus, when stripes were formed in the tectum by compound eyes, fibers of passage were seen between stripes and no segregation was observed in the optic tract (Fawcett and Willshaw, 1982).

An activity-dependent mechanism is plausible because ganglion cells from corresponding points in the two eyes see different parts of the visual world and therefore fire asynchronously, whereas ganglion cells close together in the same eye see the same part of the visual world and should fire in a closely correlated manner (Willshaw and von der Malsburg, 1976). The spontaneous activity from neighboring cells with the same type of receptive field is also positively correlated ( $\Lambda$ rnett, 1978). Fibers firing in synchrony and terminating on the same postsynaptic cell may produce EPSP summation and facilitation. Other fibers terminating on the same cell but not firing in synchrony would not show facilitation and EPSP summation. If summation increases the effectiveness of synapses and more effective synapses are preferentially retained (Hebb, 1949;
Changeux and Danchin, 1976; Willshaw and von der Malsburg, 1976; Schmidt and Edwards, 1983), then synapses from neighboring cells would stabilize one another. Since the number of synaptic sites per postsynaptic cell does not increase when excess presynaptic fibers occupy an area of tectum (Murray et al., 1982), then this type of model predicts not only that synapses from neighboring cells would stabilize one another, but also that they would destabilize synapses from the other eye. This destabilization should occur since their occupation of some of a limited number of sites would prevent the other eye from achieving maximum effectiveness.

The effect of citrate on segregation. Noninjected control fish had significantly greater patchiness in their projections than did any of the injected groups, including the fish with binocular citrate injections. There were two substances which inhibited segregation in these fish, TTX and citrate. Because TTX was shipped in dry citrate buffer solution, citrate-injected controls were used in order to distinguish between the activity-blocking effect of TTX and the influence of citrate. The citrate effect was probably not caused by trauma due to repeated fluid injections, since repeated binocular injections of very dilute TTX (and therefore very dilute citrate) do not reduce segregation (Meyer, 1982). Also, no outward signs of trauma or infection were seen. The inhibitory effect of citrate on segregation may have been caused by citrate-induced changes in activity. In a preliminary study, J. T. Schmidt (unpublished data) found that intraocular citrate injections, in the same concentrations used in this study, increased spontaneous activity without obviously changing the responsiveness to visual stimuli. Increasing the spontaneous activity of both eyes necessarily increases the probability that ganglion cells from the two eyes would fire simultaneously. This might thereby lessen the drive for segregation if segregation depends upon asynchronous activity between eyes. Since TTX blocks all activity, it would block the increased spontaneous activity and thereby cancel out any effects that citrate might have upon segregation. However, other possible mechanisms for the inhibition of segregation by citrate cannot be ruled out. If citrate does not act through increased spontaneous activity, then the citrate and TTX effects on segregation could be additive.

\section{Binocular block of activity during early segregation}

This study tested whether binocular block of activity would affect segregation when the two projections were competitively unequal; that is, one intact and the other regenerating. The binocular block drastically reduced patchiness in both projections but did not entirely prevent the development of subtle variations in density. Meyer (1982), on the other hand, used competitively equal projections (both regenerating) and concluded that binocular TTX injections totally prevented segregation. Since the TTX block did not prevent the regenerating fibers from establishing effective connections, as our electrophysiological results demonstrated, the difference between our results and Meyer's (1982) were probably not caused by differences in the initial equality of the projections. Rather, the difference in our conclusions reflects the differences in our controls, methods of producing binocular innervation, and methods of measuring segregation. Whereas Meyer (1982) deflected a single bundle of fibers, we diverted the entire projection ipsilaterally. With only a single bundle, subtle variations may have been missed. Whereas we quantified segregation in TTX fish and compared with citrate controls (accounting for the influence of citrate itself on segregation), Meyer (1982) qualitatively compared TTX fish with noninjected controls or with fish injected with dilute 'I'IX (and therefore dilute citrate). The contrast between the degree of segregation in TTX fish and uninjected fish in this study is very great and could be interpreted as a total prevention of segregation. However, the 
correct controls for TTX fish are citrate fish, and between these two a large but not total reduction can be deduced. If the effect of citrate is through increased spontaneous activity, then the stronger conclusion may be justified, but this remains to be proven.

The effect of TTX appeared to be due to the activity block rather than to damage of the retinal ganglion cells. In TTX injected fish, the retinal ganglion cells were not counted. Damage to the retinal ganglion cells due to the application of TTX itself was not likely, however. Edwards and Grafstein (1983) previously showed that 36 days of repeated TTX injections during regeneration did not reduce ganglion cell number at all when compared with citrate controls. Although some damage may have occurred in the additional 20 days of injection in this experiment, it is very unlikely that this could account for the large reduction in segregation in the TTX fish. Our test fish, blocked continuously for 6 months (more than twice as long as the experimental fish), showed only very slight reductions in ganglion cell numbers. Thus, from the early injection experiments, it can be concluded that activity blockade reduces segregation.

Parallels to ocular dominance patches in mammals. In young kittens and monkeys, the geniculocortical projections from the two eyes initially overlap, then segregate (Rakic, 1976; LeVay et al., 1980). This segregation may be blocked in cats by a binocular block of activity (Stryker, 1981) and is decremented by dark rearing (Swindale, 1981). After the TTX block is released at 8 weeks, segregation can still take place (Stryker, 1981). Similar results have been demonstrated in goldfish. Both this study and Meyer's (1982) showed that binocular block reduces or prevents segregation in fish. When the block is removed, segregation occurs (Meyer, 1982). Thus, there are strong parallels between ocular dominance patches in fish tectum and mammalian visual cortex.

These parallels occur in spite of several differences between the two systems. One difference is that the retinotectal projection is monosynaptic, whereas the pathway from retina to cortex in mammals involves a relay nucleus. Another difference is that the experimental paradigm in this study used a regenerating projection which has no exact counterpart in the mammalian visual system. In mammals a corticogeniculate projection exists for both pathways. Although a tectoretinal pathway has been described in fish (Witkovsky, 1971; Vanegas et al., 1973; Schmidt, 1979), it is largely a crossed projection, so that no tectoretinal projection should exist for the RIP in our fish. Finally, the RIP is an immature projection competing with a mature NCP. In mammals, the two projections to the cortex share the same developmental immaturity. The strong parallels, in spite of the differences, suggest that activity-dependent segregation of inputs may be a widespread phenomenon.

\section{References}

Arnett, D. W. (1978) Statistical dependence between neighboring retinal ganglion cells in goldfish. Exp. Brain Res. 32: 49-53.

Boss, V., and J. T. Schmidt (1982) Tests for a role of activity in the formation of ocular dominance patches. Soc. Neurosci. Abstr. 8: 668.

Changeux, J. P., and A. Danchin (1976) Selective stabilization of developing synapses as a mechanism for the specification of neuronal networks. Nature 264: 705-712.

Constantine-Paton, M., and M. I. Law (1978) Eye specific bands in the tecta of three-eyed frogs. Science 202: 639-64l.

Constantine-Paton, M., and M. I. Law (1978) Eye specific bands in the tecta of three-eyed frogs. Science 202: 639-641.

Easter, S. S., and J. T. Schmidt (1977) Reversed visuomotor behavior mediated by induced ipsilateral retinal projections in goldfish. $J$. Neurophysiol. 40: 1245-1254.

Easter, S. S., J. T. Schmidt, and S. M. Leber (1978) The paths and destinations of the induced ipsilateral retinal projection in goldfish. J. Embryol Exp. Morphol. 45: 145-159.

Edwards, D. L, and B. Grafstein (1983) Intraocular tetrodotoxin in goldfish hinders optic nerve regeneration. Brain Res. 269: 1-14.

Fawcett, J. W., and D. J. Willshaw (1982) Compound eyes project stripes on the optic tectum in Xenopus. Nature 296: 350-352.

Freeman, J. A., and J. Stone (1969) A technique for current sourcedensity analysis of field potentials and its application to the frog cerebellum. In Neurobiology of Cerebellar Evolution and Development, R. R. Llinàs, ed., pp. 412-443, American Medical Association, Chicago.

Hebb, D. O. (1949) Organization of Behavior, John Wiley \& Sons, Inc., New York.

Hubel, D. H., and T. N. Wiesel (1969) Anatomical demonstration of columns in the monkey striate cortex. Nature 221: 747-750.

Hubel, D. H., T. N. Wiesel, and S. LeVay (1977) Plasticity of ocular dominance columns in monkey striate cortex. Philos. Trans. R. Soc. Lond. (Biol.) 278: 377-409.

Ide, C. R., S. E. Fraser, and R. L. Meyer (1983) Eye dominance columns formed by an isogenic double nasal frog eye. Science 221: 293-295.

Law, M., and M. Constantine-Paton (1980) Right and left eye bands in frogs with unilateral tectal ablations. Proc. Natl. Acad. Sci. U. S. A. 77: 2314-2318.

Law, M., and M. Constantine-Paton (1981) Anatomy and physiology of experimentally produced striped tecta. J. Neurosci. 1: 741-759.

LeVay, S., M. P. Stryker, and C. J. Shatz (1978) Ocular dominance columns and their development in layer IV of the cat's visual cortex: A quantitative study. J. Comp. Neurol. 179: 223-244.

LeVay, S., D. H. Hubel, and T. N. Wiesel (1980) The development of ocular dominance columns in normal and visually deprived monkeys. J. Comp. Neurol. 191: 1-51.

Levine, R., and M. Jacobson (1975) Discontinuous mapping of retina into tectum innervated by both eyes. Brain Res. 98: 172-176.

Lo, R., and R. Levine (1980) Time course and pattern of optic fiber regeneration following tectal lobe removal in the goldfish. J. Comp. Neurol. 191: 295-314.

Luiten, P. G. M. (1975) The horseradish peroxidase technique applied to the teleostean nervous system. Brain Res. 89: 181-186.

Mesulam, M. -M. (1978) Tetramethyl benzidine for horseradish peroxidase neurohistochemistry: A noncarcinogenic blue reaction product with superior sensitivity for visualizing neural afferents and efferents. J. Histochem. Cytochem. 26: 106-117.

Meyer, R. L. (1982) Tetrodotoxin blocks the formation of ocular dominance columns in goldfish. Science 218: 589-591.

Murray, M., S. C. Sharma, and M. P. Edwards (1982) Target regulation of synaptic density in the compressed retinotectal projection of goldfish. J. Comp. Neurol. 209: 374-385.

Rakic, P. (1976) Prenatal genesis of connections subserving ocular dominance in the rhesus monkey. Nature 261: 467-471.

Schmidt, J. T. (1978) Retinal fibers alter tectal positional markers during the expansion of the half retinal projection in goldfish. J. Comp. Neurol. 177: 279-300.

Schmidt, J. T. (1979) The laminar organization of optic nerve fibers in the tectum of goldfish. Proc. R. Soc. Lond. (Biol.) 205: 287-306.

Schmidt, J. T., and D. L. Edwards (1983) Activity sharpens the map during the regeneration of the retinotectal projection in goldfish. Brain Res. 269: 29-39.

Schmidt, J. T., and J. A. Freeman (1980) Electrophysiologic evidence that the retinotectal project in the goldfish is nicotinic cholinergic. Brain Res. 187: 129-142.

Schmidt, J. T., D. L. Edwards, and C. A. O. Stuermer (1983) The reestablishment of synaptic transmission by regenerating optic axons in goldfish: Time course and effects of blocking activity by intraocular injection of tetrodotoxin. Brain Res. 269: 15-27.

Sharma, S. C. (1973) Anomalous retinal projections after removal of contralateral tectum in adult goldfish. Exp. Neurol. 41: 661-669.

Shatz, C. J., and M. P. Stryker (1978) Ocular dominance in layer IV of the cat's visual cortex and the effects of monocular deprivation. J. Physiol. (Lond.) 281: 267-283.

Springer, A. D., and S. M. Cohen (1981) Optic fiber segregation in goldfish with two eyes innervating one tectal lobe. Brain Res. 255: 23-36.

Stryker, M. P. (1981) Late segregation of geniculate afferents to the cat's visual cortex after recovery from binocular impulse blocade. Soc. Neurosci. Abstr. 7: 842.

Swindale, N. V. (1981) Absence of ocular dominance patches in dark reared cats. Nature 290: 332-333. 
Vanegas, H., J. Amat, and F. Fssayag-Millan (1973) Flectrophysiological evidence of tectal efferents to the fish eye. Brain Res. 54: 309 313.

Wiesel, T. N., D. H. Hubel, and D. M. K. Lam (1971) Autoradiographic demonstration of ocular dominance in the monkey striate cortex by means of transneuronal transport. Brain Res. 79: 273-279.

Willshaw, D., and C. von der Malsburg (1976) How patterned neural connections can be set up by self organization. Proc. $R$. Soc. Lond.
(Biol.) 194: 431-445.

Winer, B. J. (1962) Statistical Principles in Experimental Design, pp. 43-45, McGraw Hill Book Co., New York.

Witkovsky, P. (1971) Synapses made by myelinated fibers running to teleost and elasmobranch retinas. J. Comp. Neurol. 142: 205-221.

Wolburg, H. (1978) Growth and myelination of goldfish optic nerve fibers after retina regeneration and nerve crush. $Z$. Naturforsch. 33 . 988-996. 